\title{
Early Improvement in One Week Predicts the Treatment Response to Escitalopram in Patients with Social Anxiety Disorder: A Preliminary Study
}

\author{
Kang-Seob Oh, ${ }^{1, *}$, Eunsook Shin ${ }^{1, *}$, Juwon $\mathrm{Ha}^{2}$, Dongwon Shin', Youngchul Shin ${ }^{1}$, Se-Won Lim ${ }^{1}$ \\ ${ }^{1}$ Department of Psychiatry, Kangbuk Samsung Hospital, Sungkyunkwan University School of Medicine, Seoul, ${ }^{2}$ Department of Psychiatry, \\ Myongji Hospital, Seonam University School of Medicine, Goyang, Korea
}

\begin{abstract}
Objective: Social anxiety disorder (SAD) shows relatively delayed responses to pharmacotherapy when compared to other anxiety disorders. Therefore, more effective early therapeutic decisions can be made if the therapeutic response is predictable as early as possible. We studied whether the therapeutic response at 12 weeks is predictable based on the early improvement with escitalopram at 1 week.

Methods: The subjects were 28 outpatients diagnosed with SAD. The subjects took $10-20 \mathrm{mg} /$ day of escitalopram. The results of the Liebowitz social anxiety scale (LSAS), Hamilton anxiety rating scale, and Montgomery-Asberg depression rating scale were evaluated at 0, 1, 4, 8, and 12 weeks of treatment. Early improvement was defined as a $\geq 10 \%$ reduction in the LSAS total at 1 week of treatment, and endpoint response was defined as a $\geq 35 \%$ reduction in the LSAS total score. The correlation between clinical characteristics and therapeutic responses was analyzed by simple linear regression. The correlation between early improvement responses and endpoint responses was analyzed by multivariate logistic regression analysis and receiver operating characteristic curves.

Results: When we adjusted the influence of a $\geq 35 \%$ reduction in the LSAS total endpoint score on a $\geq 10 \%$ reduction of the LSAS total score at 1 week of treatment for the patients' age, the early improvement group at 1 week of treatment was expected to show stronger endpoint responses compared to the group with no early improvement.

Conclusion: The results suggest that a $\geq 10 \%$ reduction in the LSAS total score in a week can predict endpoint treatment response.
\end{abstract}

KEY WORDS: Social phobia; Escitalopram; Drug therapy.

\section{INTRODUCTION}

Social anxiety disorder (SAD) is one of the most common anxiety disorders. The National comorbidity survey reported that the lifetime prevalence of SAD was $12.1 \%{ }^{1)}$ This chronic condition affects $3-13 \%$ of people in the United States at some time during their life. SAD has a median age of onset of 13 years. ${ }^{2)}$ Lifetime major comorbid disorders were present in $69 \%$ of subjects with SAD. ${ }^{3)}$ Because of these characteristics, SAD can lead to significant functional impairment in patients. Selective serotonin reuptake inhibitors (SSRIs) are currently widely

\footnotetext{
Received: July 30, 2015 / Revised: September 25, 2015

Accepted: October 5, 2015

Address for correspondence: Se-Won Lim, MD, PhD

Department of Psychiatry, Kangbuk Samsung Hospital,

Sungkyunkwan University School of Medicine, 29 Saemunan-ro,

Jongno-gu, Seoul 03181, Korea

Tel: +82-2-2001-2214, Fax: +82-2-2001-2211

E-mail: knappe@paran.com

*These two authors contributed equally to this work
}

prescribed for this common, long-lasting, and sometimes disabling anxiety disorder.

The stability of the SSRI treatment, in addition to their safety, tolerability, and ability to treat comorbid conditions, supports their use as a first-line treatment. ${ }^{4)}$ Paroxetine was the first SSRI indicated for the treatment of SAD, and its efficacy has been clearly proven in 12-week, randomized, placebo-controlled studies. ${ }^{5,6}$ Citalopram, fluvoxamine, and sertraline may also be safe and effective treatments for SAD. ${ }^{7-9)}$ In addition, escitalopram has shown significant efficiency in SAD. ${ }^{7-9)}$ In these studies of $\mathrm{SAD}$, the therapeutic response is usually become evident at 12 weeks, which is slow in comparison to response times for major depressive disorder, panic disorder, and generalized anxiety disorder. ${ }^{10)}$ Prolonged time lag between initiation of treatment and response is expected to be associated with decreased treatment compliance of patients and/or failure of treatment. Therefore, more effective early therapeutic decisions can be made if

(a) This is an Open-Access article distributed under the terms of the Creative Commons Attribution Non-Commercial License (http://creativecommons.org/licenses/by-nc/4.0) which permits unrestricted non-commercial use, distribution, and reproduction in any medium, provided the original work is properly cited. 
the therapeutic response occurs as early as possible.

A few studies on SAD have addressed the prediction of treatment outcome. Versiani et al. ${ }^{11)}$ found that alcohol abuse, depression, and anxiety were associated with treatment outcome. Chambless et al. ${ }^{12)}$ reported that depression, patients' expectations of treatment, personality disorder traits, severity of impairment, and frequency of negative thoughts during social interactions were predictors. Studies of gabapentin reveal that an age $>35$ years predicted response to treatment. ${ }^{13)}$ One study of fluvoxamine found that higher heart rate and blood pressure, as well as elevated baseline measures of anxiety and depression, appeared to predict nonresponse. ${ }^{14)}$ A placebo-controlled study of paroxetine found that greater baseline severity appeared to predict better response. ${ }^{15)}$ Another study of paroxetine in SAD found that duration of treatment was a significant predictor of treatment response. ${ }^{16)}$ A study of sertraline found that patients with later-onset SAD tend to have a better response to treatment than earlier-onset patients. $^{17)}$

In other anxiety disorders, early improvement (Hamilton anxiety rating scale [HAMA] improvement) during duloxetine treatment of generalized anxiety disorder predicts response and remission at endpoint. ${ }^{18)}$ A Clinical Global Impression Improvement (CGI-I) score at week 2 was a reliable predictor of achieving endpoint response for pregabalin and venlafaxine in generalized anxiety disorder. ${ }^{19)}$ In a treatment study of panic disorder, improvement as reflected in CGI-I ratings and change from baseline in the HAMA at weeks 1, 2, and 3 significantly predicted endpoint clinical remission. ${ }^{19)}$ However, no studies have indicated that early treatment response can predict the final treatment response in SAD.

In summary, across studies examining a variety of epidemiologic factors and medication types, there are fewer consistent predictors of treatment response in SAD than there are in other anxiety disorders. In addition, the value of early improvement has not been established for treatment with SSRIs. The authors thought that it would be very helpful for clinicians to be able to predict as early as possible the treatment response to SSRIs at 12 weeks. In usual clinical practice, outpatients usually return for a follow-up visit after one week. Therefore, 1 week after SSRI treatment is the earliest time point at which to observe and predict the response of given treatment in typical clinical practice. Previous studies about other anxiety disorders have reported that improvement at 1 week can predict endpoint remission. ${ }^{19)}$ Considering the slow response time of $\mathrm{SAD}$, we hypothesized that the one-week early improve- ment criteria would demonstrate a $10 \%$ reduction from baseline. Therefore, the aim of this study was to identify whether the therapeutic response at the 12 weeks is predictable at one-week follow-up based on the early improvement due to administration of escitalopram.

\section{METHODS}

We recruited individuals with SAD who were 18-65 years of age from a hospital-based outpatient clinic. Thirty-five individuals who met the Diagnostic and Statistical Manual for Mental Disorders 4th edition $(\mathrm{DSM}-\mathrm{IV})^{20)}$ criteria for SAD were initially screened. All patients were assessed using the Mini International Neuropsychiatric Interview, ${ }^{21)}$ and a principle diagnosis of SAD was independently confirmed by two psychiatrists.

Patients with other axis-I disorders within the last six months according to DSM-IV, including schizophrenia or other psychotic disorders, or if they had a prominent risk of functional impairment due to an axis II disorder were excluded. We excluded patients with a currently high depression score of $\geq 18$ on the Hamilton depression rating scale (HAMD) ${ }^{22)}$ Finally twenty-eight clients met criteria for SAD. Participants gave written informed consent before enrollment. The protocol of this study was approved by the institutional review board at Kangbuk Samsung Hospital in Seoul, Korea (C08078).

\section{Measures}

The primary outcome measure was the Liebowitz social anxiety scale (LSAS), which provides a symptom severity score of fear and avoidance in social situations. The scale has been translated and standardized in Korean. ${ }^{23)}$ Secondary outcome measures were the HAMA, ${ }^{24)}$ which measures symptom severity of anxiety, and the Montgomery-Asberg depression rating scale (MADRS), ${ }^{25)}$ which assesses the symptom severity of depression. All outcome measures were assessed at baseline, 1, 4, 8, and 12 weeks.

\section{Treatment}

The study was a 12-week open trial of escitalopram treatment. Escitalopram was initiated at $10 \mathrm{mg}$ per day. Subsequent dose increases up to $20 \mathrm{mg}$ per day were based on tolerability and clinical response.

Efficacy measures of early improvement were defined as a $\geq 10 \%$ reduction from baseline in LSAS total or subscale score at 1 week. The endpoint response was defined as a $\geq 35 \%$ reduction from baseline in the LSAS total score. 


\section{Statistical Methods}

Demographic and clinical variables were analyzed by the Mann-Whitney U-test for continuous variables and the Fisher's Exact Test for categorical variables at baseline. Baseline differences in demographic and clinical characteristics were compared between early improvement and endpoint response. Correlation of the difference in clinical rating scale between the endpoint and baseline was performed by linear regression analysis. In addition, the clinical rating scale between the patients with early improvement and non-improvement at 1 week and between the responders and non-responders at the endpoint (12 week) were compared by linear regression analysis. Examination of early improvement as a predictor of endpoint response was analyzed by receiver operating characteristic (ROC) analyses. Statistical analysis using the last observation carried forward method was performed by PASW Statistics software, version 18.0 (IBM Co., Armonk, NY, USA). The cut-off for statistical significance was set at $p<0.05$.

\section{RESULTS}

\section{Demographic and Descriptive Sample Characteristics}

A total of 28 patients participated. The demographic characteristics are presented in Table 1. Participants were $18-62$ years of age (mean age, $43.25 \pm 2.38$ years). The mean age of onset was $22.14( \pm 14.68)$ years, and most patients had an early-onset disorder. Most patients were male (78.6\%). We evaluated SAD subtypes according to the DSM-IV; 17 (60.7\%) patients were the generalized type and $11(39.3 \%)$ patients were the non-generalized type.

When measuring baseline clinical anxiety, the baseline mean of the LSAS total score, the fear subscale score of LSAS, and the avoidance subscale score of the LSAS were $72.75 \pm 33.22,37.75 \pm 16.03$, and $35.00 \pm 18.02$, respectively. The mean of the HAMA total score, the psychological subscale score of HAMA, and the somatic subscale score of HAMA were $11.57 \pm 8.04,8.32 \pm 6.43$, and $3.25 \pm 2.50$, respectively, and the mean of the MADRS was 6.42 \pm 7.27 . When the subjects were divided into groups based on early improvement, there were no significant differences in demographic characteristics and clinical efficacy measures between patients with and without early improvement. When the subjects were divided into groups based on the endpoint response ( $\geq 35 \%$ reduction in LSAS total score), the mean age of responders was lower than the mean age of non-responders (responders: mean 37 years, range 30.5-41.5 years; non-responders: mean 49 years, range 43.0-56.0 years). No significant correlations were

Table 1. Demographic and clinical characteristics of patients $(n=28)$

\begin{tabular}{lc}
\hline \multicolumn{1}{c}{ Variable } & Data \\
\hline Age $(y r)$ & $43.25 \pm 2.379$ \\
Onset age $(y r)$ & $22.14 \pm 14.677$ \\
Sex, male & $22(78.6)$ \\
Education $(y r)$ & $14.57 \pm 2.379$ \\
LSAS & $72.75 \pm 33.222$ \\
Fear subscale & $37.75 \pm 16.031$ \\
Avoidance subscale & $35.00 \pm 18.020$ \\
HAMA & $11.57 \pm 8.038$ \\
Psychological subscale & $8.32 \pm 6.429$ \\
Somatic subscale & $3.25 \pm 2.503$ \\
MADRS & $6.42 \pm 7.269$ \\
\hline
\end{tabular}

Values are presented as mean \pm standard deviation or number (\%). LSAS, Liebowitz social anxiety scale; HAMA, Hamilton anxiety rating scale; MADRS, Montgomery-Asberg depression rating scale.

Table 2. Comparison of demographic and clinical characteristics at 1 week and 12 week

\begin{tabular}{|c|c|c|c|c|c|c|}
\hline \multirow{2}{*}{ Variable } & \multicolumn{3}{|c|}{ Early improvement } & \multicolumn{3}{|c|}{ Endpoint response } \\
\hline & $<10 \%(n=17)$ & $\geq 10 \%(n=11)$ & $p$ value & $<35 \%(n=15)$ & $\geq 35 \%(n=13)$ & $p$ value \\
\hline Age $(y r)^{\dagger}$ & $47(35.5-53.0)$ & $41(27.0-48.0)$ & 0.404 & $49(43.0-56.0)$ & $37(30.5-41.5)$ & $0.007^{*}$ \\
\hline Onset age $(y r)^{\dagger}$ & $23(13.0-31.0)$ & $14(10.0-21.5)$ & 0.169 & $25(13.0-45.0)$ & $14(9.0-26.0)$ & 0.111 \\
\hline Sex, male $^{\ddagger}$ & $14(82.4)$ & $8(72.7)$ & 0.653 & $12(80.0)$ & $10(76.9)$ & 0.600 \\
\hline Education $(y r)^{\dagger}$ & $15(12.0-16.0)$ & $16(14.0-16.0)$ & 0.487 & $15(13.0-16.0)$ & $16(13.0-16.0)$ & 0.496 \\
\hline LSAS & $69.00 \pm 35.35$ & $78.54 \pm 30.32$ & 0.468 & $74.40 \pm 36.42$ & $70.84 \pm 30.45$ & 0.784 \\
\hline Fear subscale & $35.00 \pm 17.53$ & $42.00 \pm 13.01$ & 0.267 & $37.46 \pm 17.82$ & $38.07 \pm 14.39$ & 0.922 \\
\hline Avoidance subscale & $34.00 \pm 18.25$ & $42.00 \pm 13.01$ & 0.722 & $36.93 \pm 19.14$ & $32.76 \pm 17.11$ & 0.552 \\
\hline HAMA & $11.70 \pm 9.18$ & $11.36 \pm 6.28$ & 0.915 & $13.00 \pm 8.39$ & $9.92 \pm 7.59$ & 0.322 \\
\hline Psychological subscale & $8.41 \pm 7.41$ & $8.18 \pm 4.85$ & 0.928 & $9.06 \pm 6.81$ & $7.46 \pm 6.10$ & 0.520 \\
\hline Somatic subscale & $3.29 \pm 2.71$ & $3.18 \pm 2.27$ & 0.910 & $3.93 \pm 2.60$ & $2.46 \pm 2.22$ & 0.123 \\
\hline MADRS & $5.64 \pm 4.97$ & $7.63 \pm 10.02$ & 0.490 & $6.86 \pm 4.88$ & $5.92 \pm 9.51$ & 0.739 \\
\hline
\end{tabular}

Values are presented as median (interquartile range), number (\%), or mean \pm standard deviation.

LSAS, Liebowitz social anxiety scale; HAMA, Hamilton anxiety rating scale; MADRS, Montgomery-Asberg depression rating scale.

One week: early improvement ( $\geq 10 \%$ reduction in the LSAS total score); 12 week: endpoint response ( $\geq 35 \%$ reduction of LSAS total score). ${ }^{*} p<0.05$; 'Mann-Whitney U-test; " Fisher's exact test. 
found in the age of onset, gender, education, and clinical measures such as LSAS, HAMA, and MADRS (Table 2).

The mean daily escitalopram dose received by each subject across the 12-week period was $10.98 \mathrm{mg}$. Only one was taking the maximum dose of $20 \mathrm{mg}$ at the treatment endpoint.

\section{Prediction of Endpoint Clinical Remission}

According to simple linear regression analysis, patients with the generalized subtype had a higher endpoint response than patients with the non-generalized subtype $(p=0.04)$. Significant correlations were found between the fear subscale of the LSAS and the endpoint response $(p=0.037)$. No other significant correlations were found in the LSAS total score, the avoidance subscale of the LSAS, HAMA, or MADRS (Table 3).

The association between early improvement measures at 1 week and the endpoint clinical response were analyzed using multivariate logistic regression models. Results from the analysis found that only a $\geq 10 \%$ reduction in the LSAS total score in 1 week remained a significant predictor of the endpoint clinical response after adjusting for age (odds ratio, 7.448). No significant associations were observed between the $\geq 10 \%$ reduction in any subscale of the LSAS and the endpoint clinical re-

Table 3. Linear regression analysis between the clinical scale at baseline and the endpoint repsonse

\begin{tabular}{crc}
\hline Variable & Coefficients & p value \\
\hline Social phobia subtype & $-20.123 \pm 9.313$ & $0.040^{*}$ \\
LSAS total & $0.274 \pm 0.142$ & 0.063 \\
Fear subscale & $0.635 \pm 0.288$ & $0.037^{*}$ \\
Avoidance subscale & $0.430 \pm 0.266$ & 0.118 \\
HAMA total & $0.387 \pm 0.621$ & 0.539 \\
Psychological subscale & $0.711 \pm 0.770$ & 0.364 \\
Somatic subscale & $-0.705 \pm 2.004$ & 0.728 \\
MADRS & $0.627 \pm 0.0681$ & 0.366
\end{tabular}

LSAS, Liebowitz social anxiety scale; HAMA, Hamilton anxiety rating scale; MADRS, Montgomery-Asberg depression rating scale. ${ }^{*} p<0.05$ sponse (Table 4).

\section{Receiver Operating Characteristic Curve}

Predicted the $\geq 35 \%$ of reduction in LSAS total scores at endpoint created from logistic regression equations for the significantly measure (the $\geq 10 \%$ reduction of the LSAS total score) were entered into ROC analyses using endpoint clinical response as the criterion (Fig. 1). The area under the ROC curve (AUC) ranged from 0.66 to 0.86 (95\% confidence interval, 0.574-0.949). Based upon previously reported ranges for evaluating the area under the curve in ROC analyses, ${ }^{26)}$ the $\geq 10 \%$ reduction of LSAS total score at 1 week was in the fair range as a predictor of endpoint clinical response.

\section{DISCUSSION}

In this study, early improvement during escitalopram treatment of SAD was examined using five efficacy measures: LSAS total score at 1 week, LSAS fear subscale score at 1 week, LSAS avoidance subscale score at 1

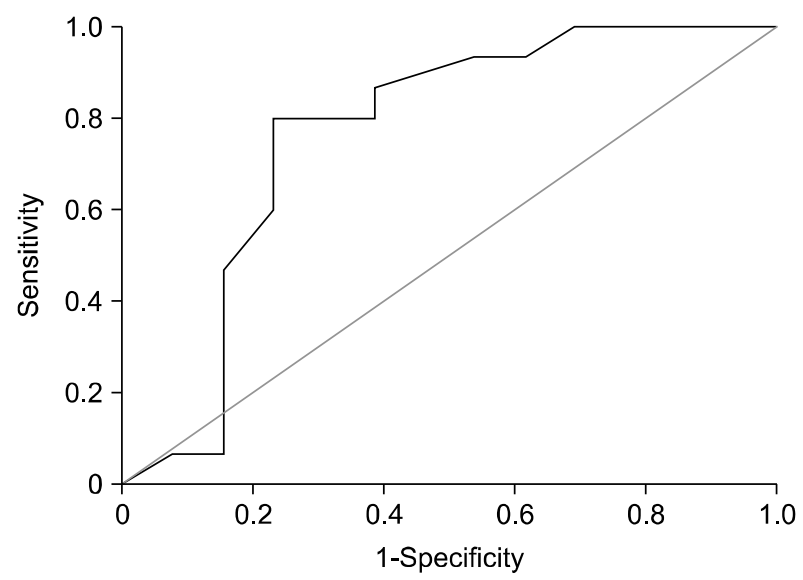

Fig. 1. Receiver-operator characteristic curve for the total Liebowitz social anxiety scale score at week 1 predicting endpoint clinical response.

Table 4. Early improvement predicting the endpoint response

\begin{tabular}{|c|c|c|c|c|c|}
\hline Week 1 & OR & $95 \% \mathrm{Cl}$ & $p$ value & Sensitivity & Specificity \\
\hline \multicolumn{6}{|l|}{ LSAS total } \\
\hline Response $10 \%$ & 7.448 & $1.019-54.449$ & $0.048^{*}$ & 0.615 & 0.706 \\
\hline \multicolumn{6}{|l|}{ LSAS fear } \\
\hline Response $10 \%$ & 5.358 & $0.773-37.119$ & 0.089 & 0.615 & 0.706 \\
\hline \multicolumn{6}{|l|}{ LSAS avoidance } \\
\hline Response $10 \%$ & 3.929 & $0.488-31.652$ & 0.199 & 0.385 & 0.600 \\
\hline
\end{tabular}

Multivariate logistic regression analysis adjusted for age.

LSAS, Liebowitz social anxiety scale; OR, odds ratio; $95 \% \mathrm{Cl}, 95 \%$ confidence interval.

${ }^{*} p<0.05$. 
week, HAMA, and MADRS. Multivariate logistic regression analyses demonstrated that a $\geq 10 \%$ reduction in LSAS total score has predictive value as early as week 1 . These results suggest that global improvement of social anxiety symptoms may be more useful in predicting the ultimate outcome in SAD than mood and anxiety measures. In our study, ROC curves were used as a visual tool to confirm early improvement predictors. In one study finding that early improvement predicts endpoint remission status in sertraline treatment of panic disorder, the ROC analysis AUC of 0.67 had significant value as a predictor. $^{27)}$ In another study of treatment predictors in generalized anxiety disorder, AUCs ranging from $0.70-0.80$ were in the fair range. ${ }^{19)}$ Based upon previously reported ranges for other anxiety disorders and the Metz's study ${ }^{26)}$ for evaluating the area under the curve in ROC analyses, the $\geq 10 \%$ reduction of LSAS total score at 1 week performed best in the fair range as a predictor of endpoint response. ROC analysis suggested that the LSAS total score at 1 week has good sensitivity and specificity.

Treatment guidelines recommend that physicians wait up to 8 weeks in major depressive disorder, 10 weeks in panic disorder, and 12 weeks in generalized anxiety disorder before switching medication due to lack of efficacy. ${ }^{7,8,28,29)}$ SSRI trial durations of approximately 12 weeks are the current recommendation in SAD. ${ }^{10)}$ Since this is a long period compared to other disorders, the initial drug selection in SAD is more important. Therefore, the ability to predict final clinical response from early response has potentially important clinical implications in SAD. For the patient showing early improvement, especially those who demonstrate a diminution in social anxiety symptoms by 1 week, the clinician can feel confident that the initial treatment is likely to be associated with a favorable outcome. By observing such early changes, the physician can avoid unnecessary upward dose titration or use of additional interventions that may increase side effects or cost, and can provide reassurance to patients that their treatment appears to be on track to a positive outcome. On the other hand, physicians may consider therapeutic alternatives for the patient displaying no early improvement in social anxiety score. Further research is needed to explore whether therapeutic strategies such as dosage change/augmentation or cognitive behavior therapy can increase the probability of remission in patients expected to remain symptomatic because of early non-response.

In SAD, the slightest hint of negativity in another's face signals danger, causing the amygdale to initiate a fear response. At the same time, the effects from elevated nor- epinephrine affect the peripheral nervous system and trigger many signs of social anxiety. Serotonin dysregulation may also cause some symptoms seen in SAD. Therefore, $\mathrm{SAD}$ and panic disorder have some neurobiology in common. For some people, a panic attack in a social setting triggers fear of social settings and eventually leads to social avoidance. Thus, the first step of treatment for SAD is a physiology assessment to control rapid heart rate and excessive stress response, or pharmacological treatment such as SSRIs and serotonin-norepinephrine reuptake inhibitors. $^{30,31)}$ We found that high fear scores on the LSAS subscale at baseline were associated with the endpoint response. For escitalopram treatment, inhibition of the fear-circuitry is faster than the response of avoidance behavior. Considering that early improvement in our study was determined at 1 week, the endpoint clinical response was more associated with the fear subscale than the avoidance subscale. Patients with generalized SAD had more comorbid psychiatric disorders, family history, and higher anxiety severity and heart rate variability than patients with non-generalized anxiety. ${ }^{29,32,33)}$ The activity of the fear-circuitry appears only in certain circumstances in non-generalized SAD, whereas in the generalized type, the fear-circuitry activity is sensitive for a wide range of social situations, which is therefore more closely linked to the biological response. This may explain why the endpoint response in generalized type was better correlated.

In our study, the predictive probability of clinical endpoint response or non-response from early improvement is not associated with depression, global anxiety symptoms, or demographic characteristics. In past studies, the most referent predictor of change between pre- and post-treatment appeared to be pretreatment depression. In Versiani et al., ${ }^{11)}$ high baseline Hamilton anxiety or depression scale scores predicted a positive outcome. As Chambless et al. $^{12)}$ noted, the initial level of depression was predictive for treatment outcome of SAD. In contrast with this study, pretreatment depression, clinician-rated severity of impairment during social interactions was predictive for treatment outcome, whereas depression had no correlation with treatment response. ${ }^{34)}$ However, we excluded subjects with comorbidities or a depression score of $\geq 18$ on the HAMD in order to control for the possible influence of clinical depression. Thus it is difficult to determine the relationship between clinical depression and treatment response of SAD. Future studies with larger numbers of subjects should focus on whether an early improvement in LSAS can predict treatment response when clinical depression coexist with SAD. 
Several important study limitations should be mentioned. First, patients were enrolled without a control group, and there was a relatively small number of patients. However, the same rater evaluated the therapeutic effect under the same conditions, and we minimized the impact of anxiety and depression. We suggest a large double-blind study of treatment predictors of SAD for further research.

Another important limitation of the current study is that criterion of early improvement of SAD was set without existing standards. However, standards for early improvement in other anxiety disorders and mood disorders have been proposed. In one study of depression, early improvement indicated an increased chance of achieving a 50\% reduction in HAMD scores by 1 week with SSRI treatment compared with placebo. ${ }^{35)}$ When using sertraline for panic disorder, improvement as reflected in CGI-I ratings and change from baseline in the HAMA at weeks 1, 2, and 3 significantly predicted endpoint clinical remission (defined as no full panic attacks and a CGI-Severity rating of 1 or 2). ${ }^{27)}$ One study of duloxetine for generalized anxiety disorder (GAD) explored early improvement on HAMA in relation to endpoint HAMA response ( $\geq 50 \%$ improvement from baseline), HAMA remission $(\leq 7)$, CGI-I response $(\leq 2)$, and functional remission as measured by the Sheehan Disability Scale Global Functional Improvement $(\leq 5){ }^{27,36)}$ Another study compared the predictive value of early response to treatment outcomes in patients with GAD treated with benzodiazepines, serotonin receptor (5HT-1A) partial agonists, or a placebo. Categories of response at weeks 1 and 2 were defined by the HAMA total score. ${ }^{36)}$ In study of early improvement predictors when treating generalized anxiety disorder with pregabalin or venlafaxine $X R$, early improvement was defined as a $\geq 20 \%$ reduction from baseline in the HAMA total score at week 1 or $2 .{ }^{19)}$ However, there is little research linking early improvement to treatment outcome in SAD. Therefore, there was no available standard for early improvement in SAD treatment. The authors set an early improvement criterion as a $10 \%$ reduction in total LSAS score from baseline because, when we consider the slow response time of SAD, the $20 \%$ reduction criterion that is used in other anxiety disorders is unlikely to be achieved in 1 week. Because the authors of this study intended to determine whether early improvement can predict treatment outcome after 1 week, we did not measure the treatment response at week 2 . This might be a major limitation of this study.

The endpoint clinical responses to treatment of SAD were defined differently in many studies. In Liebowitz et $a l .{ }^{37)}$ the treatment efficacy of paroxetine for generalized
SAD was evaluated with the mean change from baseline in the LSAS total score and with the proportion of patients exhibiting a therapeutic response (defined as a CGI-I score of 1 or 2 ). In a randomized controlled trial of venlafaxine extended release in generalized SAD, the treatment response was defined as a CGI-I score of 1 or 2 and the definitions of remission were used as an LSAS total score $\leq 30$ and a CGI-I score of $1{ }^{38)}$ In a study examining the efficacy of escitalopram in treatment resistant patients with $\mathrm{SAD}$, late responders were defined as patients with a $35 \%$ decrease in the LSAS total score or subscale score at the endpoint or 12 weeks compared to baseline. ${ }^{39)}$ In this study, the treatment response at the endpoint was defined as a $35 \%$ reduction in LSAS total score in 12 weeks. Nevertheless, the results may appear different depending on criteria of early or endpoint response, and further work is needed to evaluate optimal early and remission thresholds.

In summary, early improvement, as reflected in the LSAS total score improvement, predicted endpoint response for social anxiety patients treated with escitalopram. The current results suggest a connection between early improvement and endpoint response that can be used to guide clinical decision-making. Treatment efficacy and compliance would be enhanced by use of an early improvement criterion.

\section{- Acknowledgments}

We gratefully acknowledge financial support from $\mathrm{H}$. Lundbeck A/S, Copenhagen-Valby, Denmark for this work. Lundbeck was not responsible for creation of the study protocol, the data analysis, data interpretation, or writing of the manuscript.

\section{REFERENCES}

1. Kessler RC, Stein MB, Berglund P. Social phobia subtypes in the National Comorbidity Survey. Am J Psychiatry 1998; 155:613-619.

2. Kessler RC, Berglund P, Demler O, Jin R, Merikangas KR, Walters EE. Lifetime prevalence and age-of-onset distributions of DSM-IV disorders in the National Comorbidity Survey Replication. Arch Gen Psychiatry 2005;62:593-602.

3. Schneier FR, Johnson J, Hornig CD, Liebowitz MR, Weissman MM. Social phobia. Comorbidity and morbidity in an epidemiologic sample. Arch Gen Psychiatry 1992;49: 282-288.

4. Blanco C, Raza MS, Schneier FR, Liebowitz MR. The evidence-based pharmacological treatment of social anxiety disorder. Int J Neuropsychopharmacol 2003;6:427-442.

5. Lydiard RB, Bobes J. Therapeutic advances: paroxetine for the treatment of social anxiety disorder. Depress Anxiety 2000;11:99-104.

6. Stein MB, Chartier MJ, Hazen AL, Kroft CD, Chale RA, 
Coté $\mathrm{D}$, et al. Paroxetine in the treatment of generalized social phobia: open-label treatment and double-blind placebo-controlled discontinuation. J Clin Psychopharmacol 1996; 16:218-222.

7. Stein MB, Fyer AJ, Davidson JR, Pollack MH, Wiita B. Fluvoxamine treatment of social phobia (social anxiety disorder): a double-blind, placebo-controlled study. Am J Psychiatry 1999;156:756-760.

8. Van Ameringen MA, Lane RM, Walker JR, Bowen RC, Chokka PR, Goldner EM, et al. Sertraline treatment of generalized social phobia: a 20-week, double-blind, placebocontrolled study. Am J Psychiatry 2001;158:275-281.

9. Varia IM, Cloutier CA, Doraiswamy PM. Treatment of social anxiety disorder with citalopram. Prog Neuropsychopharmacol Biol Psychiatry 2002;26:205-208.

10. Bandelow B, Baldwin DS, Dolberg OT, Andersen HF, Stein DJ. What is the threshold for symptomatic response and remission for major depressive disorder, panic disorder, social anxiety disorder, and generalized anxiety disorder? $J$ Clin Psychiatry 2006;67:1428-1434.

11. Versiani M, Amrein R, Montgomery SA. Social phobia: long-term treatment outcome and prediction of response--a moclobemide study. Int Clin Psychopharmacol 1997;12:239254.

12. Chambless DL, Tran GQ, Glass CR. Predictors of response to cognitive-behavioral group therapy for social phobia. $J$ Anxiety Disord 1997;11:221-240.

13. Pande AC, Davidson JR, Jefferson JW, Janney CA, Katzelnick DJ, Weisler RH, et al. Treatment of social phobia with gabapentin: a placebo-controlled study. J Clin Psychopharmacol 1999; 19:341-348.

14. Slaap BR, van Vliet IM, Westenberg HG, Den Boer JA. Responders and non-responders to drug treatment in social phobia: differences at baseline and prediction of response. J Affect Disord 1996;39:13-19.

15. Montgomery SA. Implications of the severity of social phobia. J Affect Disord 1998;50 Suppl 1:S17-S22.

16. Stein DJ, Stein MB, Pitts CD, Kumar R, Hunter B. Predictors of response to pharmacotherapy in social anxiety disorder: an analysis of 3 placebo-controlled paroxetine trials. J Clin Psychiatry 2002;63:152-155.

17. Van Ameringen M, Oakman J, Mancini C, Pipe B, Chung H. Predictors of response in generalized social phobia: effect of age of onset. J Clin Psychopharmacol 2004;24:42-48.

18. Pollack MH, Kornstein SG, Spann ME, Crits-Christoph P, Raskin J, Russell JM. Early improvement during duloxetine treatment of generalized anxiety disorder predicts response and remission at endpoint. J Psychiatr Res 2008;42:11761184.

19. Baldwin DS, Schweizer E, Xu Y, Lyndon G. Does early improvement predict endpoint response in patients with generalized anxiety disorder (GAD) treated with pregabalin or venlafaxine XR? Eur Neuropsychopharmacol 2012;22: 137-142.

20. American Psychiatric Association; Task Force on DSM-IV. Diagnostic and statistical manual of mental disorders: DSM-IV. Washington, DC:American Psychiatric Association; 1994.

21. Lecrubier Y, Sheehan DV, Weiller E, Amorim P, Bonora I, Sheehan $\mathrm{KH}$, et al. The Mini International Neuropsychiatric Interview (MINI). A short diagnostic structured interview: reliability and validity according to the CIDI. European Psychiatry 1997;12:224-231.
22. Williams JB. A structured interview guide for the Hamilton Depression Rating Scale. Arch Gen Psychiatry 1988;45:742747.

23. Yu ES, Ahn CI, Park KH. Factor structure and diagnostic efficiency of a Korean version of the Liebowitz social anxiety scale. Korean J Clin Psychol 2007;26:251-270.

24. Shear MK, Vander Bilt J, Rucci P, Endicott J, Lydiard B, Otto MW, et al. Reliability and validity of a structured interview guide for the Hamilton Anxiety Rating Scale (SIGH-A). Depress Anxiety 2001;13:166-178.

25. Ahn YM, Lee KY, Yi JS, Kang MH, Kim DH, Kim JL, et al. A validation study of the Korean-version of the MontgomeryAsberg depression rating scale. J Korean Neuropsychiatr Assoc 2005;44:466-476.

26. Metz CE. Basic principles of ROC analysis. Semin Nucl Med 1978;8:283-298.

27. Pollack MH, Rapaport MH, Fayyad R, Otto MW, Nierenberg AA, Clary CM. Early improvement predicts endpoint remission status in sertraline and placebo treatments of panic disorder. J Psychiatr Res 2002;36:229-236.

28. van Vliet IM, den Boer JA, Westenberg HG. Psychopharmacological treatment of social phobia; a double blind placebo controlled study with fluvoxamine. Psychopharmacology (Berl) 1994;115:128-134.

29. Stein MB, Chavira DA. Subtypes of social phobia and comorbidity with depression and other anxiety disorders. $J$ Affect Disord 1998;50 Suppl 1:S11-S16.

30. Stahl SM. Stahl's essential psychopharmacology: neuroscientific basis and practical applications. Cambridge: Cambridge University Press;2013.

31. Wehrenberg M, Prinz S. The anxious brain: the neurobiological basis of anxiety disorders and how to effectively treat them. New York:W.W. Norton \& Co.;2007.

32. Boone ML, McNeil DW, Masia CL, Turk CL, Carter LE, Ries BJ, et al. Multimodal comparisons of social phobia subtypes and avoidant personality disorder. J Anxiety Disord 1999;13:271-292.

33. Levin AP, Saoud JB, Strauman T, Gorman JM, Fyer AJ, Crawford R, et al. Responses of "generalized" and "discrete" social phobics during public speaking. J Anxiety Disord 1993; 7:207-221.

34. Scholing A, Emmelkamp PM. Prediction of treatment outcome in social phobia: a cross-validation. Behav Res Ther 1999;37:659-670.

35. Taylor MJ, Freemantle N, Geddes JR, Bhagwagar Z. Early onset of selective serotonin reuptake inhibitor antidepressant action: systematic review and meta-analysis. Arch Gen Psychiatry 2006;63:1217-1223.

36. Rynn M, Khalid-Khan S, Garcia-Espana JF, Etemad B, Rickels K. Early response and 8-week treatment outcome in GAD. Depress Anxiety 2006;23:461-465.

37. Liebowitz MR, Stein MB, Tancer M, Carpenter D, Oakes $\mathrm{R}$, Pitts CD. A randomized, double-blind, fixed-dose comparison of paroxetine and placebo in the treatment of generalized social anxiety disorder. J Clin Psychiatry 2002;63:66-74.

38. Liebowitz MR, Mangano RM, Bradwejn J, Asnis G; SAD Study Group. A randomized controlled trial of venlafaxine extended release in generalized social anxiety disorder. $J$ Clin Psychiatry 2005;66:238-247.

39. Pallanti S, Quercioli L. Resistant social anxiety disorder response to Escitalopram. Clin Pract Epidemiol Ment Health 2006;2:35. 